\title{
Documents Clustering Using K-Means Algorithm
}

\author{
R.B. Wahyu*, Arnold Vito Ezra Panjaitan \\ President University \\ *Corresponding author: rbw0101@gmail.com
}

\begin{abstract}
Nowadays in the digital era, people could easily access and stored a wide range of information through the Internet into documents. With the huge number of unstructured documents with various type of information in digital storage, people need an application that could help them organize and classify the documents automatically. Documents Clustering using K-Means Algorithm is a desktop-based documents clustering application which implement K-Means Algorithm to provides clustering output based on the documents content similarity up to $85 \%$ accuracy based on the user expectation.
\end{abstract}

\section{INTRODUCTION}

With the ease of getting various kind of information in this digital era from the Internet, people start to live with paperless environment not buying newspapers, magazines or books. Many of the information stored from the Internet will be useful for further occasion when it is needed such as for research, literature study, supporting ideas, or references. These various collections of information needed to be categorized to make them easily accessed based on their contents.

Although there have been several clustering applications developed, none has been clustering the documents and then grouped the documents into folders based on the documents category as accurate as possible. The existence of such application will greatly help people in classifying their scattered documents into a set of folders which contains similar information one to another internally, but substantially different with other folders.

The major problems are: 1. Currently there is no such application that implement the clustering results into grouping the documents into folders 2 . Retrieve the information of each documents, 3. Implement K-Means Algorithm for clustering the documents, 4 . The accuracy of clustered documents using K-Means Algorithm. These can be solved by having an application to clusters and categorized the documents using K-Means Algorithm.

The objectives of this research are:
- To create a desktop-based documents clustering application using K-Means Algorithm application

- To implement the clustering result from K-Means Algorithm into grouping the documents into folders based on their contents similarity

Limitations

This application uses an English stop words collection; thus, the application could only read documents which its content is in English.

All documents to be processed must be in text format such as .txt, .pdf and .doc documents.

The clustering result does not guarantee 100\% accuracy based on the user expectation because the application will merely learn and clustered the documents based on the documents content similarity one to another.

Other limitations are: the processing time is heavily depending on the number of documents, the documents content length and number of clusters given.

\section{METHODS}

This section explains the methods used in the development of the application.

\section{$1.1 \quad$ Clustering}

Clustering is an unsupervised learning technique to group objects based on their distance and/or similarity. It is called as an unsupervised learning technique because clustering let the machine (computer or program) learns mere from objects given, then the machine will automatically categorize these objects into which group [19].

In clustering, we only specify the rule of clustering method of how the machine will compute the distance between objects and compute the distance between clusters. The purpose of performing clustering technique is to know how the objects will be categorized based on the rule given to the machine. 
IT FOR SOCIETY, Vol. 03, No. 02

ISSN 2503-2224

Table 1 Clustering vs Linear Analysis [15]

\begin{tabular}{|l|l|l|}
\hline \multicolumn{1}{|c|}{ About } & \multicolumn{1}{|c|}{ Clustering } & \multicolumn{1}{c|}{$\begin{array}{c}\text { Linear Discriminant } \\
\text { Analysis }\end{array}$} \\
\hline Other name & Unsupervised learning & Supervised learning \\
\hline $\begin{array}{l}\text { Training or } \\
\text { learning period }\end{array}$ & $\begin{array}{l}\text { Object category is unknown, } \\
\text { rule of classification is given } \\
\text { (generalized distance based) }\end{array}$ & Object category is known \\
\hline Purpose of training & $\begin{array}{l}\text { To know the category of each } \\
\text { object }\end{array}$ & $\begin{array}{l}\text { To know the classification } \\
\text { rule }\end{array}$ \\
\hline $\begin{array}{l}\text { After training } \\
\text { (usage) }\end{array}$ & $\begin{array}{l}\text { To classify object into a } \\
\text { number of categories }\end{array}$ & $\begin{array}{l}\text { To classify object into a } \\
\text { number of categories }\end{array}$ \\
\hline
\end{tabular}

\subsection{Document Clustering}

Documents clustering is an action of collecting the similarities of documents contents, then automatically categorized those documents with their contents similarities. Its goal is to learn automatically the category of every documents then create clusters that similar internally but substantially different with the other clusters [7].

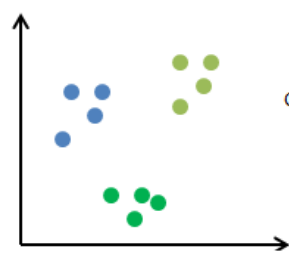

Scattered Document

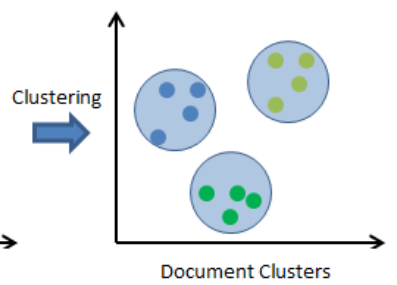

Figure 1 Documents Clustering Illustration [9]

\subsection{K-Means Algorithm}

K-Means algorithm is a type of unsupervised learning algorithm developed by J. Mac Queen (1967) to find groups for a set of data based on their attribute with the number of groups as a cluster represented by variable K [20]. Variable K representing a positive integer number which is used to determine how many cluster's centroids will be used to classify the data, in which the centroid represent the value of the center of each cluster.

Generally, k-means algorithm works by taking a unique attribute from each data given as a data point, then assign these data points to the nearest cluster centroid, after that the centroids will begin moving to better fit the clusters themselves. These data points and centroids will keep moving until the algorithm find the minimum fitting error between the data sets and centroids by updating the centroids to be the mean value of the clusters repeatedly in each loop until no movement needed anymore [23].

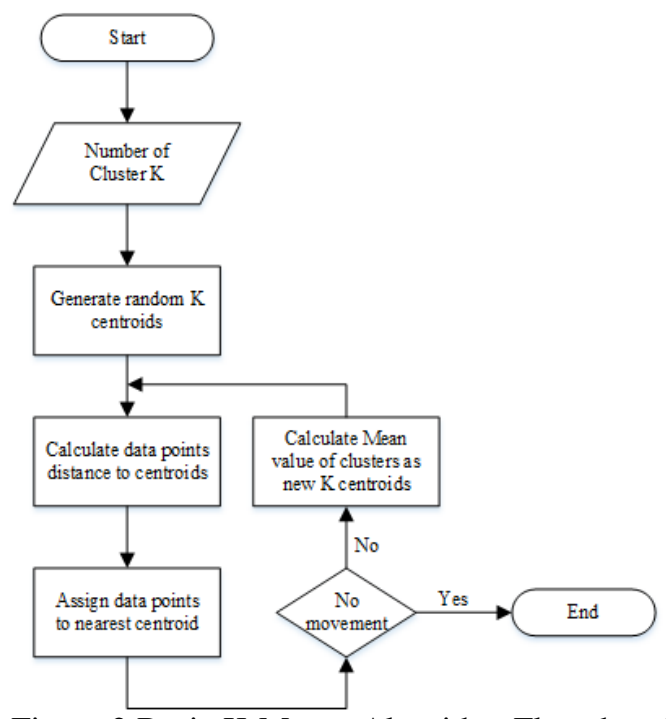

Figure 2 Basic K-Means Algorithm Flowchart [18]

1.4 Information Retrieval

Information retrieval is an activity of obtaining relevant information from a large amount of information databases [6]. Information retrieval help the algorithm to identify the relevant information that represent each document. By having the relevant information representation of each document, the document clustering algorithm will be able to provide a qualified clustering output of the documents.

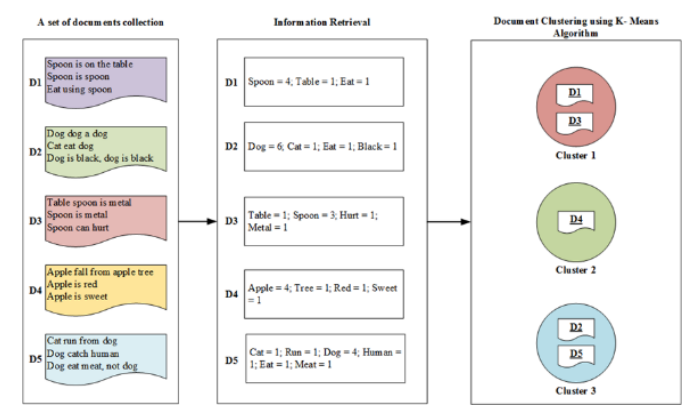

Figure 3 Information Retrieval role in Document Clustering

1.5 Terms Frequency (TF) - Inverse Document Frequency (IDF)

Terms Frequency (TF) is the numerical measurement of how frequent a word appears in a document [22], which will show how important a word to a document.

$$
T F_{(t)}=\log _{10} \frac{\text { Number of } t \text { appearence in a document }}{\text { document length }}
$$

Inverse Document Frequency (IDF) is the statistical weight measurement of how important a word to a set of documents collection [22]. IDF diminishes the weight of words that appears very frequently in 
a set of documents collection, but it scales up the rare ones.

$$
I D F_{(t)}=\frac{\text { Total documents in the collection }}{\text { Number of documents with word } t \text { in it }}
$$

Basically, the ways to get the relevant information of each document using Information Retrieval method is by getting the TF-IDF value of each document information. After having the TF-IDF of each word among the documents, the documents will be represented as a mutual comparable vector with the intention to have the numerical model of each document. Each document vector will be represented using the TF-IDF result of each term (word) from each document [23].

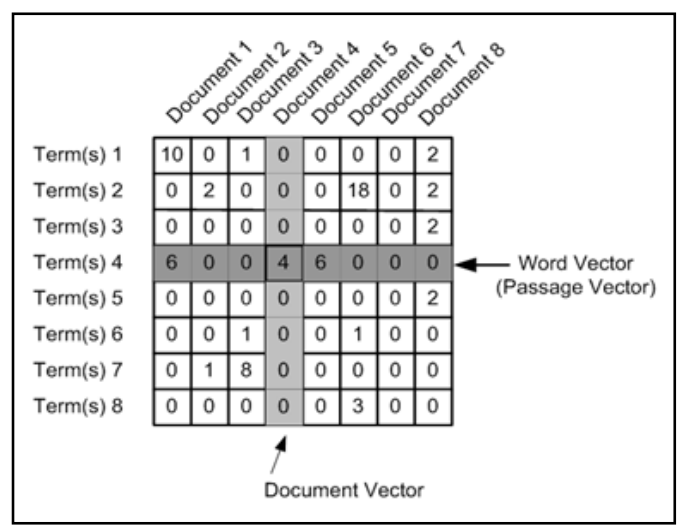

Figure 4 TF-IDF vector space model [12]

\section{EXPERIMENTAL RESULTS}

Tests are done in order to evaluate the effectiveness of the proposed methods in previous section. Test results are described in tables below:

Table 2 K-Means Performance 1st Evaluation

\begin{tabular}{|l|l|}
\hline Documents to be processed & 15 documents \\
\hline Documents content length & Up to 1000 words \\
\hline Processed time & 5 minutes 55 seconds \\
\hline Clusters to be produced & 4 clusters \\
\hline Expected result & $\begin{array}{l}\text { Cluster 1:5 documents about Article 1 } \\
\text { Cluster 2: } 3 \text { documents about Article 2 } \\
\text { Cluster 3:5 documents about Article 3 } \\
\text { Cluster 4: } 2 \text { documents about Article 4 }\end{array}$ \\
\hline Result & $\begin{array}{l}\text { Cluster 1:5 documents about Article 1 } \\
\text { Cluster 2:3 documents about Article 2 } \\
\text { Cluster 3:5 documents about Article 3 } \\
\text { Cluster 4:2 documents about Article 4 }\end{array}$ \\
\hline Result accuracy evaluation & $\frac{5+3+5+2}{15} \times 100 \%=100 \%$ \\
\hline
\end{tabular}

Table 3 K-Means Performance 2nd Evaluation

\begin{tabular}{|l|l|}
\hline Documents to be processed & 22 documents \\
\hline Documents content length & Up to 1000 words \\
\hline Processed time & 17 minutes 23 seconds \\
\hline Clusters to be produced & 5 clusters \\
\hline \multirow{5}{*}{ Expected result } & $\begin{array}{l}\text { Cluster 1: } 7 \text { documents about Article 1 } \\
\text { Cluster 2: } 2 \text { documents about Article 2 } \\
\text { Cluster 3: } 3 \text { documents about Article 3 } \\
\text { Cluster 4: } 5 \text { documents about Article 4 } \\
\text { Cluster 5: } 5 \text { documents about Article 4 }\end{array}$ \\
\hline Result & $\begin{array}{l}\text { Cluster 1: } 6 \text { documents about Article 1 } \\
\text { Cluster 2: } 2 \text { documents about Article 2 } \\
\text { Cluster 3:3 documents about Article 3 } \\
\text { Cluster 4: 4 documents about Article 4 } \\
\text { Cluster 5:3 documents about Article 5 }\end{array}$ \\
\hline Result accuracy evaluation & $\frac{6+2+3+4+3}{22} \times 100 \%=81,81 \%$ \\
\hline
\end{tabular}

Table 4 K-Means Performance 3rd Evaluation

\begin{tabular}{|l|l|}
\hline Documents to be processed & 30 documents \\
\hline Documents content length & Up to 200 words \\
\hline Processed time & 7 minutes 41 seconds \\
\hline Clusters to be produced & 4 clusters \\
\hline Expected result & $\begin{array}{l}\text { Cluster 1: } 5 \text { documents about Article 1 } \\
\text { Cluster 2: 10 documents about Article 2 } \\
\text { Cluster 3: 7 documents about Article 3 } \\
\text { Cluster 4: 8 documents about Article 4 }\end{array}$ \\
\hline Result & $\begin{array}{l}\text { Cluster 1:5 documents about Article 1 } \\
\text { Cluster 2:5 documents about Article 2 } \\
\text { Cluster 3:5 documents about Article 3 } \\
\text { Cluster 4: } 8 \text { documents about Article 4 }\end{array}$ \\
\hline Result accuracy evaluation & $\frac{5+5+5+8}{30} \times 100 \%=76,67 \%$ \\
\hline
\end{tabular}

Based on the Evaluation data of the K-Means Algorithm performance on Table 2 to Table 4, this application could produce $\frac{100+81,81+76,67}{3} \times 100 \%=86,16 \%$ accuracy.

Table 5 Documents Clustering Results Evaluation

\begin{tabular}{|l|l|l|l|}
\hline No & Scenario & Expected Result & Evaluation \\
\hline 1 & $\begin{array}{l}\text { Number of clustering } \\
\text { iteration }\end{array}$ & $\begin{array}{l}\text { The total iteration of } \\
\text { the clustering process is } \\
\mathrm{k}^{*}(\mathrm{k} * 500) \text { times } \\
\text { (where } \mathrm{k} \text { is number of } \\
\text { clusters) }\end{array}$ & As expected \\
\hline 2 & Clustering output result & $\begin{array}{l}\text { The number of folders } \\
\text { group produced by the } \\
\text { system is based on } \\
\text { number of clusters } \\
\text { assigned }\end{array}$ & As expected \\
\hline 3 & Total files information & $\begin{array}{l}\text { The total files clustered } \\
\text { should be equals to the } \\
\text { total files processed by } \\
\text { the application }\end{array}$ & As expected \\
\hline 4 & $\begin{array}{l}\text { Total files and total words } \\
\text { information }\end{array}$ & $\begin{array}{l}\text { Total files and total } \\
\text { words value should } \\
\text { restart from } 0 \text { when the } \\
\text { user start another } \\
\text { clustering process to a } \\
\text { different directory }\end{array}$ & As expected \\
\hline
\end{tabular}

\section{DISCUSSION}

This section presents discussion on why Documents Clustering using K-Means Algorithm uses the methods it uses.

- $\quad$ Clustering 
Documents Clustering using K-Means Algorithm utilizes clustering method for classifying the objects rather than another classifying the objects method such as Linear Discriminant Analysis.

The advantage of this method is:

System could maximize the classification result without needed to have a specific training example.

\section{- K-Means Algorithm}

This application uses K-Means Algorithm to calculate the documents similarity one to another.

The advantage of this method is:

K-Means algorithm works by taking unique attribute from each data, then assigning these attributes to the nearest cluster to better fit the clusters themselves by finding the minimum fitting error between the data sets and centroids by updating the centroids to be the mean value of the clusters.

\section{CONCLUSIONS}

First, Document Clustering using K-Means Algorithm application is a desktop-based application developed to classify text documents purely based on its content similarity.

Second, the clustering result of the documents does not guarantee $100 \%$ accuracy compared to the user expectation. The accuracy of this application is up to $85 \%$ compared to user expectation because the classification process is really depending on the number of documents, number of clusters, the document contents, and the documents length.

In the future, Documents Clustering using KMeans Algorithm will have user interface enhancement and an advanced documents processing method and the results itself.

\section{REFERENCES}

[1] Ambler, S. W. (n.d.). Agile Modeling. Retrieved March 15 2017, from UML 2 Use Case Diagrams: AN Agile Introduction: http://www.agilemodeling.com/artifacts/useC aseDiagram.htm

[2] Erb, E. (n.d.). Github. Retrieved April 20, 2017, from Document Clustering Program in Java: https:/github.com/ezraerb/DocumentCluster

[3] File: K Means Example Step 1.svg. (n.d.). Retrieved March 20, 2017, from Wikipedia: https://en.wikipedia.org/wiki/File:K_Means_E xample_Step_1.svg

[4] File: K Means Example Step 2.svg. (n.d.). Retrieved March 20, 2017, from Wikipedia: https://en.wikipedia.org/wiki/File:K_Means_E xample_Step_2.svg
[5] File: K Means Example Step 3.svg. (n.d.). Retrieved March 20, 2017, from Wikipedia: https://en.wikipedia.org/wiki/File:K_Means_E xample_Step_3.svg

[6] Information Retrieval. (n.d.). Retrieved March 22, 2017, from http://www.doc.ic.ac.uk/ nd/surprise_97/jour nal/vol4/hks/inf_ret.html

[7] Jajoo, P. (2008). Document Clustering. Retrieved February 5, 2017

[8] K-Means Clustering. (n.d.). Retrieved February 4, 2017, from A Tutorial on Clustering Algorithms: https://home.deib.polimi.it/matteucc/Clusterin g/tutorial_html/kmeans.html

[9] Kunwar, S. (n.d.). Text Documents Clustering using K-Means Algorithm. Retrieved October 20, 2016, from Code Project: https://www.codeproject.com/Articles/439890 /Text-Documents-Clustering-using-K-MeansAlgorithm

[10] Osinski, S., \& Weiss, D. (n.d.). Carrot2. Retrieved April 27, 2017, from Carrot2 Search: http://search.carrot2.org/stable/search

[11] Osinski, S., \& Weiss, D. (n.d.). Carrot2 User and Developer Manual. Retrieved April 27, 2017, from Carrot2: http://download.carrot2.org/head/manual/inde x.html\#chapter.introduction

[12] Rose, B. (n.d.). Document Clustering with Python. Retrieved March 19, 2017, from http://brandonrose.org/clustering

[13] Shah, N., \& Mahajan, S. (2012). Document Clustering: A Detailed Review. International Journal of Applied Information Systems (IJAIS). Retrieved February 6, 2017

[14] Sousa, S. d. (n.d.). The Advantages and Disadvantages of RAD Software Development. Retrieved October 4, 2016, from Susan de Sousa's My PM Expert: www.my-project-managementexpert.com/the-advantages-anddisadvantages-of-rad-softwaredevelopment.html

[15] Teknomo, K. (n.d.). Difference of Cluster Analysis and Discriminant Analysis. Retrieved February 2, 2017, from Revoledu: http://people.revoledu.com/kardi/tutorial/LDA /Cluster\%20and\%20discriminant\%20analysis. html

[16] Teknomo, K. (n.d.). Discriminant Analysis Tutorial. Retrieved February 2, 2017, from Revoledu: http://people.revoledu.com/kardi/tutorial/LDA I

[17] Teknomo, K. (n.d.). Euclidean Distance. Retrieved March 23, 2017, from Revoledu: http://people.revoledu.com/kardi/tutorial/Simi larity/EuclideanDistance.html 
[18] Teknomo, K. (n.d.). How the K-Mean Clustering algorithm works? Retrieved March 1, 2017, from Revoledu: http://people.revoledu.com/kardi/tutorial/kMe an/Algorithm.htm

[19] Teknomo, K. (n.d.). What is Clustering? Retrieved January 30, 2017, from Revoledu: http://people.revoledu.com/kardi/tutorial/Clust ering/clustering.htm

[20] Teknomo, K. (n.d.). What is K-Mean Clustering? Retrieved January 31, 2017, from Revoledu:
http://people.revoledu.com/kardi/tutorial/kMe an/WhatIs.htm

[21] toletol, K. (n.d.). Rapid Application Development (RAD) Model. Retrieved October 6, 2016, from Wikipedia: https://en.wikipedia.org/wiki/File:RADModel. JPG

[22] What does tf-idf mean? (n.d.). Retrieved March 23, 2017, from http://www.tfidf.com/

[23] Zong, J. (n.d.). K Means Clustering with Tfidf Weights. Retrieved March 10, 2017, from http://jonathanzong.com/blog/2013/02/02/kmeans-clustering-with-tfidf-weights 